\title{
Peut-on parler de l'origine du langage ?
}

La question qui donne son titre à ma communication risque de passer pour paradoxale, voire peut-être provocatrice, en tout cas mal venue dans le colloque qui nous réunit. Il a pour titre «Fabriques de la langue ». Certes, cette formule ne se confond pas, par le sens, avec « Origine du langage ». Mais les deux expressions sont en rapport étroit : la notion de « Fabriques de la langue » semble bien avoir quelque relation avec le problème de l'« Origine du langage ». Et ce n'est évidemment pas un hasard si plusieurs auteurs de communications ont prévu de poser le problème de l'origine du langage, sans, à ce qu'il semble, éprouver la moindre angoisse à l'idée de l'aborder.

Mais il y a plus grave encore, sans doute. Non contente d'être paradoxale et provocatrice, ma question risque en outre de passer pour complètement inutile, peut-être même stupide. Pour une raison qui apparaît immédiatement : depuis très longtemps on lui a donné une réponse. Une réponse affirmative, bien sûr. L'origine du langage est même l'un des premiers problèmes touchant le langage à avoir été traité. Je rappelle, rapidement, que la Bible, non contente de le traiter, le résout. Dans le texte biblique, la création du langage se trouve partagée entre Dieu et sa création, l'homme. C'est naturellement Dieu qui a le premier mot :

« Dieu dit : " Que la lumière soit !" Et la lumière fut. Dieu vit que la lumière était bonne ; et Dieu sépara la lumière d'avec les ténèbres. Dieu appela la lumière jour, et il appela les ténèbres nuit. Ainsi il y eut un soir et il y eut un matin ; ce fut le premier jour » (Genèse, chapitre I, 3-5).

Texte complexe, on le voit. La profération du nom lumière dans un contexte performatif suffit pour créer la lumière, et la séparer des ténèbres, qui, elles, existaient sans avoir à être nommées. Mais, sitôt créée, la lumière reçoit de Dieu un autre nom, jour, ainsi que les ténèbres, qui reçoivent le nom de nuit. Ces nouveaux noms n'éliminent pas les anciens. Ils les complètent en instituant l'opposition périodique du jour et de la nuit.

Cependant, ce n'est pas Dieu qui a le dernier mot. Une fois la création terminée, c'est à l'homme tout frais créé que revient la tâche de la dénomination des êtres vivants, qui restent encore dépourvus de noms :

« L'Eternel Dieu forma de la terre tous les animaux des champs et tous les oiseaux du ciel, et il les fit venir vers l'homme pour voir comment il les appellerait, et afin que tout être vivant portât le nom que lui donnerait l'homme. Et l'homme donna des noms à tout le bétail, aux oiseaux du ciel et à tous les animaux des champs; mais pour l'homme, il ne se trouvait point d'aide qui fût semblable à lui » (Genèse, II, 19-20)

S'ensuit la création de la femme à partir d'une côte d'Adam préalablement en- 
dormi. Et c'est de nouveau Adam qui se charge de donner à la femme son nom : « celle-ci s'appellera d'un nom qui marque l'homme, parce qu'elle a été prise de l'homme » (Genèse, II, 23).

Deux traits à remarquer particulièrement dans cette belle mise en scène de l'origine du langage. D'une part la création du langage se fait en deux temps. Elle est d'abord contemporaine de la création de l'Univers par Dieu. Les deux créations en viennent même à se confondre : il est difficile de décider si Dieu nomme en créant ou crée en nommant. Mais il ne prend pas la peine de nommer les êtres vivants : il laisse ce soin à l'homme, Adam. Les mots se répartissent donc en deux classes, selon leur origine, divine ou humaine. Second trait important : le texte de la Genèse ne parle que d'une classe de mots. Qu'ils soient créés par Dieu ou par Adam, ce sont exclusivement des noms, au sens « sémantique » du terme, c'est-à-dire des désignations d'objets. Renan en 1849 puis en 1858, dans son De l'origine du langage, en fera la remarque d'une façon extrêmement lucide:

« Il n'est question dans le passage de la Genèse que d'une certaine classe de mots et non du langage en général ; on expliquerait tout au plus par ce passage la formation du dictionnaire, mais non celle de la grammaire » (1858: 84).

Qu'on se rassure : je passe au Déluge, et, surtout, à ses suites en matière de réflexion sur le langage. Le problème de son origine est sans doute l'un de ceux qui ont donné lieu au plus grand nombre de travaux de la part des philosophes, des linguistes, des anthropologues et de quelques autres. Les ouvrages récents publiés sur le problème fournissent des bibliographies incroyablement fournies, qui continuent à s'enrichir de jour en jour. Je ne me hasarde même pas à une énumération de noms, et me contente de remarquer que le titre De l'origine $d u$ langage, avec les variantes imposées par les différences de langues et d'époques, est sans doute l'un de ceux qui ont été le plus souvent pratiqués depuis qu'on écrit des livres et des articles. Je me contente de rappeler qu'il a été utilisé en français notamment par Rousseau et Renan, en allemand par Herder et Grimm. J'ajoute aux derniers deux auteurs allemands, moins connus sans doute, mais importants pour les éléments qu'ils ont fournis à Freud sur le problème de l'origine du langage dans ses rapports avec l'inconscient. Il s'agit de Carl Abel, l'homme des sens opposés des mots primitifs, et de Hans Sperber, l'homme de l'origine sexuelle du langage : on sait l'influence profonde et durable qu'ils ont exercée, surtout pour le premier, sur les positions de Freud relatives aux relations entre langage et inconscient (voir notamment Arrivé 2008). L'inventaire des titres - on en aura une petite idée dans la bibliographie d'Auroux 2006, qu'il donne comme volontairement très lacunaire - serait hallucinant si on entreprenait de l'établir de façon exhaustive.

Tous les auteurs que j'ai cités, et bien d'autres encore, tombent générale- 
ment d'accord avec le texte biblique au moins sur un point : oui, il est possible d'aborder, peut-être même de résoudre, le problème de l'origine du langage. Faut-il donc que je me plie à leur autorité ? Et que j'interrompe en ce point mon exposé ? Peut-être pas. Car certains faits, moins massifs, sans doute, semblent montrer que la question que je me hasarde à poser n'est pas, en dépit de son caractère paradoxal, aussi inutile que la rumeur générale le laisse entendre.

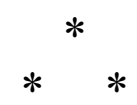

Je cite d'abord un premier fait. Il est au plus haut point remarquable par son caractère exceptionnel. Je vous le livre sous la forme littérale qui lui a été donnée en 1866. Les membres de la Société de Linguistique de Paris, qui vient de se créer, se donnent des Statuts dont l'article 2 est ainsi rédigé :

« La Société n'admet aucune communication concernant soit l'origine du langage, soit la création d'une langue universelle »

On le voit : c'est le même problème sous deux aspects qui est écarté par les linguistes de l'époque : celui de la genèse. Genèse du langage, d'une part, - enfouie dans un passé inaccessible - genèse, d'autre part, éventuelle et future, d'une langue universelle, nécessairement artificielle, qui ne pourrait être construite que selon des modes qui n'ont jamais été ceux des langues dites « naturelles ». Les membres de la Société de linguistique avaient des raisons historiques, à certains égards presque anecdotiques, qui ont été décryptées par Gabriel Bergounioux, notamment dans Hombert, ed., 2005. On peut aussi, à mon sens surtout, voir dans leur geste la manifestation de ce que j'appellerais volontiers une prudence épistémologique : le langage et les langues sont ce qu'ils sont, il est impossible de tenir un discours scientifique tant sur la façon dont ils sont devenus ce qu'ils sont que sur la façon d'en construire de nouveaux exemples. Il convient donc non pas d'interdire l'examen de ces problèmes - c'est un geste très difficile, sans doute même impossible - mais de les écarter du champ scientifique.

Je ne quitte pas cet épisode illustre de l'histoire de la Société de Linguistique sans apporter, il est vrai de façon interrogative, la précision suivante : ce geste formalisé d'exclusion d'un problème du champ de la scientificité est, à ma connaissance, un phénomène très rarement observé. Une enquête, peut-être trop brève ou trop superficielle, ne m'en a fait repérer que deux autres exemples : le refus opposé par l'Académie des sciences, dès 1775, à toute communication portant sur la quadrature du cercle et sur le mouvement perpétuel, d'ailleurs sans doute pas exactement dans les mêmes conditions. Sauf erreur, cette disposition reste en vigueur à l'Académie des Sciences, alors que la Société de Linguistique de Paris a, dès 1876, reformulé ses statuts d'une façon telle que 
l'exclusion des communications sur l'origine du langage n'est plus explicite. En tout cas elle n'est plus observée. Elle ne l'a à vrai dire jamais été rigoureusement.

Mais il n'est pas nécessaire d'interdire ou d'écarter l'examen d'un problème pour ne pas avoir à le traiter. Il suffit de dire qu'il ne se pose pas. C'est à ce traitement qu'est soumis le problème de l'origine du langage chez certains linguistes ou philosophes du langage. En dépit du titre qu'il donne à son ouvrage - De l'origine du langage, que j'ai déjà cité - c'est ce que fait Renan :

«C'est donc un rêve d'imaginer un premier état où l'homme ne parla pas, suivi d'un autre état où il conquit l'usage de la parole. L'homme est naturellement parlant, comme il est naturellement pensant, et il est aussi peu philosophique d'assigner un commencement voulu au langage qu'à la pensée » (1858:91-92).

J'attire votre attention sur l'expression un commencement voulu, où le participe adjectif voulu est à prendre, le contexte est totalement explicite, dans le sens de « conscient ». Renan le dit sans la moindre ambiguïté quelques lignes plus bas :

« D'une part, la parole est l'œuvre de l'homme et des forces qui résident en lui ; de l'autre, rien de réfléchi, rien de combiné artificiellement dans le langage, non plus que dans l'esprit. Tout y est l'œuvre des forces internes de la nature humaine, agissant sans conscience - c'est Michel Arrivé qui souligne - et comme sous l'impression vivante de la Divinité » (ibid. :92).

Je l'avoue : ce beau texte me plonge dans la plus totale perplexité. Au point que j'en viens à me poser une question apparemment insolite, une de plus : la Divinité ne serait-elle pas le nom que Renan donne à l'inconscient ? Relisez sa phrase, et écoutez celle-ci :

«Le véritable auteur des œuvres spontanées de la conscience, c'est la nature lui-même, ou, si on l'aime mieux, la cause supérieure de la nature. À cette limite il devient indifférent d'attribuer la causalité à Dieu ou à l'homme » (ibid. : 94)

... ou celle-ci, peut-être plus explicite encore :

« Partout c'est le Dieu caché, la force infinie, qui, agissant en l'absence ou durant le sommeil de l'âme individuelle, produit ces merveilleux résultats, et défie la science de comprendre ce que la nature a produit sans effort » (ibid. : 90)

Serais-je vraiment trop imprudent en avançant que « le Dieu caché, la force infinie ", c'est, peut-être, le nom que Renan donne à ce qu'il ne peut pas, ou ne veut pas nommer l'inconscient?

Mais je laisse de côté le problème théologico-linguistico-psychanalytique que pose le discours de Renan. Je me contente de remarquer qu'il confère une 
contemporanéité absolue au langage et à la pensée :

« Le langage étant la forme expressive de la pensée, l'un et l'autre doivent être tenus pour contemporains » (ibid. : 92)

De ce fait, il procède donc au renvoi dans le « rêve » de l'origine du langage, ou, mais pour lui cela revient au même, le ramène au problème de l'origine de la pensée. Sur ce point, il anticipe sur un autre auteur, que je vais convoquer dans quelques instants : Ferdinand de Saussure, qui tiendra sur ce point des propos très voisins de ceux de Renan. Cette convergence est étonnante, compte tenu de la divergence qui s'observe généralement en matière de langage entre les deux auteurs.

Il faut toutefois préciser que Renan ne réussit pas à soutenir sa position de façon aussi totalement cohérente que le fera Saussure. En effet, il tient sur deux problèmes connexes des propos qui entrent en contradiction forte avec la position générale qu'il adopte sur le problème de l'origine. Ces deux problèmes sont les suivants :

1. Le problème de la connexion du langage avec la voix. Sur ce point, Renan tient ferme pour la naturalité et la nécessité de cette connexion : «Le besoin de signifier au dehors ses pensées et ses sentiments est naturel à l'homme : tout ce qu'il pense, il l'exprime intérieurement et extérieurement. Rien non plus d'arbitraire dans l'emploi de l'articulation [au sens vocal du terme, MA] comme signe des idées. Ce n'est ni par une vue de convenance ou de commodité, ni par imitation des animaux que l'homme a choisi la parole pour formuler et communiquer sa pensée, mais bien parce que la parole est chez lui naturelle, et quant à sa production organique, et quant à sa valeur expressive » (ibid. : 90). Renan ne semble pas s'aviser qu'en posant cette relation nécessaire entre langage et parole, il pose du même coup les problèmes, litigieux et difficiles à résoudre, de la genèse de la parole humaine, illustrés aujourd'hui notamment par des questions telles que celles de la position du larynx ou du diamètre du canal hypoglosse. Les habitués du problème savent la place que ces données occupent dans les travaux contemporains sur l'origine du langage.

2. Le problème de la relation entre les mots et les choses. Renan, en dépit de ce qu'on lui a vu dire dans sa critique du texte biblique, semble bien se situer le plus souvent dans le cadre d'une conception nomenclaturiste les termes nomenclature et nomenclateur sont fréquents dans son discours - du langage. Les mots désignent les choses d'une façon directe et immédiate. Ils ont été créés en vue d'exercer cette fonction de désignation. De ce fait il arrive souvent qu'ils ressemblent à ces choses : c'est qu'ils ne sont «que l'écho de la nature dans la conscience humaine » 
(ibid. : 136). Certes, cette ressemblance écholalique a souvent été effacée par le temps. Cependant, poursuit-il, « certains idiomes conservent encore le souvenir des procédés qui présidèrent à leur création. Dans les langues sémitiques et dans l'hébreu en particulier, la formation par onomatopée est très-sensible pour un grand nombre de racines, et pour celles-là surtout qui portent un caractère marqué d'antiquité et de monosyllabisme » (ibid. : 137). Le terme onomatopée est doublement caractérisé : d'abord par son sens étymologique - « fabrique du mot», qui porte la trace du nomenclateur -, ensuite par le sens spécialisé qu'il a pris dès le grec, puis en latin et dans les langues modernes : « fabrique de mots imitateurs des choses qu'ils désignent ». Renan lui donne une place centrale dans sa théorie du langage, sans, à ce qu'il semble, se rendre compte de la contradiction qu'elle introduit à tout instant dans son discours, puisque, de la même façon que le texte biblique qu'il critique, elle réduit le langage à un « dictionnaire ».

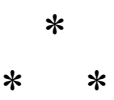

J'en viens donc à Saussure. D'abord pour préciser que, contrairement à Renan, il n'étudie pas le problème de l'origine du langage de façon continue ni systématique. Il se contente de remarques éparses à différents moments de sa réflexion. Cependant, quelle que soit leur époque, elles restent totalement homogènes entre elles quant à leur contenu.

Contrairement à ce qui a été parfois dit, Saussure n'évacue pas d'emblée le problème de l'origine du langage. Il ne récuse pas l'idée d'une humanité non pourvue du langage. Par là il introduit le problème de l'origine du langage. Sa position ne se confond donc pas avec celle, qu'il connaît, nécessairement, de la Société de Linguistique de Paris. On verra même plus loin qu'il en vient, en un point généralement occulté par les commentateurs, à examiner les conditions qui permettraient d'étudier le problème. Pour l'instant il se contente du constat suivant :

« La nature nous donne l'homme organisé pour le langage articulé, mais sans langage articulé » (2002:178).

L'expression " sans langage articulé » est à la fois explicite et ambiguë. Explicite, puisqu'elle pose le problème du passage d'un état de l'humanité dépourvue de langage à un état où elle en dispose : c'est précisément le problème de l'origine. Ambiguë, en raison de l'adjectif articulé, deux fois de suite appliqué au nom langage. Car cet adjectif est ambigu, Saussure ira même, en 1891, jusqu'à le qualifier "d'obscur et très vague », et fera " toutes réserves » sur son emploi (2002 : 145). Mais comme on voit il ne laisse pas de l'employer. Il faut 
donc essayer de lever cette " obscurité » de l'adjectif, comme Saussure le fait lui-même dans un passage du Cours III (214-215) :

1. Il peut, d'une part, signifier que le langage est soumis à l'articulation, c'est-à-dire à la division en unités de diverses dimensions et de divers statuts, par exemple les phonèmes, les mots et les syntagmes. Saussure donne souvent ce sens, de la façon la plus explicite, au syntagme langage articulé et au nom articulation, par exemple dans le $C L G$, pp. 26 (c'est un passage du Cours III), puis 156 (cette fois c'est un passage du Cours II). D'une façon particulièrement claire et vigoureuse, Martinet, plus tard, donnera à cette propriété du langage le nom de « double articulation », qui, je le remarque, donne lieu très souvent à des incompréhensions graves de la part de certains chercheurs qui, sans formation linguistique suffisante, croient pouvoir être en mesure d'aborder le problème des origines du langage. Par charité, je n'en dis pas plus long. Mais je tiens quelques références à la disposition des curieux.

2. D'autre part, Saussure utilise aussi l'expression langage articulé avec le sens qu'elle a à la fois dans l'usage quotidien et dans celui d'un grand nombre de linguistes. Le langage articulé, dans ce second sens, c'est le langage tel qu'il est manifesté par la voix humaine et les " articulations », au sens d'émissions de sons, qu'elle produit. À mon sens, c'est, dans le passage cité, le second sens, le sens profane, en quelque sorte, qui apparaît. C'est, je pense, ce qu'induit le contexte, et notamment la phrase suivante : «L'individu, organisé pour parler, ne pourra utiliser son appareil que par la communauté qui l'environne » (ibid.) Les deux termes parler et appareil font allusion à la réalisation vocale du langage. Cette interprétation est encore appuyée par le commentaire qui est donné du terme articulation (2002 : 236-237). Il n'est, en ce point, que sous-entendu que cette réalisation vocale n'est que l'une de celles qui sont possibles, exactement sur un pied d'égalité.

Ainsi, Saussure pose le sujet humain comme « organisé pour le langage ». De quelle façon va-t-il traiter la question de son accès effectif à la pratique du langage ? Il convient de distinguer deux moments :

1. Le premier est de caractère négatif. Il consiste à dénier l'existence autre qu' " idéale », " mythique »- on est tout près du « rêvé » de Renan - du " premier jour où une société humaine a parlé ». Naturellement, ce geste de dénégation s'accompagne, comme chez Renan, du rejet total de la tradition biblique et de tout ce qui lui ressemble dans les discours tenus sur l'origine du langage, c'est-à-dire à peu près tout, selon Saussure, car il a l'esprit très large en matière de critique : "Au chapitre sémiologie : la 
plupart des conceptions que se font, ou que du moins offrent les philosophes du langage font songer à notre premier père Adam appelant près de lui les divers animaux et leur donnant à chacun leur nom ». (2002:230). Saussure revient en plusieurs points sur cette dénégation de la réalité du problème de l'origine. Ainsi dans une note Item restée inédite jusqu'à 2002 : «Item. Quelle question d'origine ? - Origine de la langue. Rien ne prouve mieux la nullité de toute recherche sur l'origine de la langue. Mais sur cette question, il ne faut pas se borner aux constatations négatives. Ce qui prouve l'absence d'une question philosophique de l'origine de la langue, CE N'EST PAS UN FAIT NEGATIF, c'est le fait positif que dès le premier moment un signe ne vaut que si [ ] » (2002: 93). Hélas, ici, le texte s'interrompt, comme il est fréquent dans ces réflexions à la fois improvisées et angoissées auxquelles se livre Saussure. Et il est évidemment aventureux d'essayer de restituer les réflexions qu'il n'a pas osé écrire lui-même. Je m'y hasarde cependant, compte tenu à la fois du verbe valoir et du que si qui inaugure la formule inachevée : Saussure pensait à la valeur différentielle et négative du signe, qui ne « vaut» que dans le système d'oppositions dans lequel il s'insère, système qui suppose évidemment la pluralité déjà instituée des signes. Ce qui suffit à exclure la pertinence du geste adamique, répété, selon Saussure, par les "philosophes du langage », de donation des noms, un par un et isolément, aux objets du monde. - La composante négative de la réflexion de Saussure se poursuit en plusieurs points. Immédiatement après la réflexion que nous venons de lire, on trouve une belle métaphore géographique - Saussure en est friand - qui assimile la langue à un ruisseau : «Item. Regarder la langue et se demander à quel moment précis une telle chose a " commencé " est aussi intelligent que de regarder le ruisseau de la montagne et de croire qu'en remontant on trouvera l'endroit précis où il a sa source. Des choses sans nombre établiront qu'à tout moment le RUISSEAU existe pendant qu'on dit qu'il naît, et réciproquement il ne fait que naître pendant qu'on [ ]» (2002 : 94).Une fois encore Saussure a laissé sa phrase inachevée, elle est cependant sans doute plus facile à compléter : j'en laisse le soin à mes auditeurs.

2. Tel est donc l'aspect négatif de la réflexion de Saussure sur l'origine du langage et des langues. On a cependant aperçu que l'aspect positif a commencé à se faire jour, discrètement lors de la métaphore du ruisseau - « la langue existe pendant qu'on dit qu'elle naît» - et presque explicitement lors de l'allusion à la valeur du signe. On trouve en plusieurs autres points des éléments à proprement parler positifs, quoique souvent affectés de quelques touches énigmatiques. Ainsi on le voit donner le titre « Origine du langage » à un fragment des « Notes pour un livre sur la linguistique générale »: «Origine du langage. Inanité de la question pour 
qui prend une juste idée de ce qu'est un système sémiologique et de ses conditions de vie [ le mot est souligné par Saussure ] avant de considérer ses conditions de genèse. Il n'y a aucun moment où la genèse diffère caractéristiquement de la vie du langage, et l'essentiel est d'avoir compris la vie » (2002: 228). Comme à l'habitude chez Saussure, il faut prendre garde au sens des mots. Le terme vie, qu'il souligne lui-même, prend ici le sens de « fonctionnement, mise en acte ». L'idée est que les procédés mis en œuvre dans le fonctionnement hic et nunc du langage sous la forme de telle ou telle langue ne diffèrent pas fondamentalement des procédés qui en ont déterminé la genèse. - D'une façon plus explicite, Saussure précise qu'il n'y a pas à distinguer la «production » de la « reproduction ». Pour marquer l'originalité de sa position, Saussure l'oppose, polémiquement, par l'adverbe aujourd'hui, à l'école allemande de Franz Bopp et de ses successeurs, avec laquelle il a des rapports ambigus, le plus souvent hostiles : «Aujourd'hui, on voit qu'il y a réciprocité permanente [ entre langue et langage ] et que dans l'acte de langage la langue tire à la fois son application et sa source unique et continuelle et que le langage est à la fois l'application et le générateur continuel de la langue, la production et la reproduction » (2002: 129). Ce texte est pour une part énigmatique, certes. On aperçoit cependant que l'« acte de langage » tel qu'il est effectué par « le sujet parlant» est à la fois la mise en œuvre de la langue - c'est ce que Saussure nomme ici l'" application »- et sa source unique et continuelle. La production et le reproduction sont indistinguables. En d'autres termes, la création n'est jamais qu'une transformation et, inversement, « une transformation, c'est une création 》 (2002 : 160). - Saussure décline ces propositions de plusieurs façons. Dans la seconde conférence de linguistique générale qu'il donne à Genève en 1891, il revient sur le problème de la relation entre création et transformation : « C'est une idée très fausse que de croire que le problème de l'origine du langage soit un autre problème que celui de ses transformations » (2002 : 159). C'est en ce point qu'apparaît, de façon à mon sens unique dans sa réflexion, une allusion aux conditions qui donneraient une validité au problème de l'origine. Unique, certes, mais suffisante pour montrer que le problème n'a pas été évacué d'emblée :

« Ce serait un autre problème si l'on supposait que d'autres forces ont agi autrefois dans le langage, dont nous ne pouvons nous faire aucune idée d'après ce qui se passe aujourd'hui quand nous parlons, mais cette supposition est aussi arbitraire qu'invraisemblable ; elle revient à attribuer à l'humanité primitive des facultés ou des sens essentiellement différents de ceux que nous possédons » (2002:159)

La condition nécessaire pour poser effectivement le problème de l'origine serait donc de supposer une « humanité primitive » fondamentalement dif- 
férente de l'humanité actuelle. C'est ce que Saussure considère comme « aussi arbitraire qu'invraisemblable ». Je remarque au passage que c'est ce geste « aussi arbitraire qu'invraisemblable » que font certains chercheurs contemporains, par exemple Jean-Louis Dessales, qui ne le considèrent évidemment ni comme " arbitraire », ni comme « invraisemblable ». Dessales conclut son article de 2006, significativement intitulé « Du protolangage au langage : modèle d'une transition » de la façon suivante :

« En formant des prédicats, en les exprimant par des énoncés simples, puis par des énoncés moléculaires pour atteindre une détermination poussée des objets du discours, nos ancêtres ont pu affirmer des relations et surtout remettre en question les relations affirmées par les autres. Conformément à ce qui est généralement admis, nous avons tout lieu de penser que cette transition correspond à l'avènement d'homo sapiens » (2006: 150).

On voit comment cette position contemporaine s'éloigne de celle de Saussure. Car " l'avènement d'homo sapiens » présuppose une « humanité primitive » douée de facultés différentes de celles de l'humanité actuelle. En sorte que le protolangage évoqué par Dessales aurait des propriétés fondamentalement différentes du langage. C'est précisément le geste de Dessales que Saussure se refuse à faire. Et, bien sûr, il est parfaitement possible de contester ce refus. Il y a des tas de gens qui le font aujourd'hui, par exemple Dessales lui-même, par le silence qu'il observe, ou, de façon explicite, mais, hélas, très mal informée, Robert, 2010. Mais ce qu'on conteste alors, c'est l'entier de la conception saussurienne du langage, dont on ne peut séparer le discours qu'il tient sur l'origine.

Le discours de Saussure se poursuit donc de façon désormais parfaitement cohérente. Dans une des nouvelles notes Item de 2002, il neutralise l'opposition entre naissance et croissance : "On peut discuter éternellement sur cette " naissance ", mais son plus grand caractère, c'est d'être parfaitement le même que sa “croissance" " (2002 : 94). "L'origine du langage »? Le problème s'en confond avec celui de «l'âge des langues »:

« Toutes les langues se parlant à la même époque sont de même âge ; en ce sens qu'elles remontent à un passé égal. Il n'est pas nécessaire de déterminer la longueur de ce passé. Si l'on veut, c'est l'origine du langage, mais sans remonter aux périodes inaccessibles » $(2002: 155)$.

Et j'ai réservé pour la fin la forme la plus ferme de cette attitude, celle qui lui a été donnée, après la mort de Saussure, par les éditeurs de 1916 du Cours de linguistique générale : " En fait, aucune société ne connaît et n'a jamais connu la langue autrement que comme un produit hérité des 
générations précédentes et à prendre tel quel. C'est pourquoi la question de l'origine du langage n'a pas l'importance qu'on lui attribue généralement. Ce n'est même pas une question à poser [Je remarque ici que les éditeurs ont légèrement forcé l'expression de Saussure, en lui faisant frôler l'interdiction. Dans le Cours II Saussure semble avoir réellement dit, plus sereinement : "Cette question n'existe même pas " (Engler : 160)] ; le seul objet réel de la linguistique, c'est la vie normale et régulière d'un idiome déjà constitué » (1916-1922: 105)

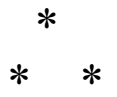

Cette position sur le problème de l'origine du langage et des langues n'est pas spécifique à Saussure. On a aperçu tout à l'heure qu'elle avait été tenue, quelques dizaines d'années auparavant, par Renan, quoique, nous l'avons vu, dans un contexte théorique bien différent. Elle est également tenue, dans un contexte plus proche de Saussure, par Victor Henry, dans ses Antinomies linguistiques, à cette différence près qu'il formule explicitement l'interdiction que Saussure évite (1896:25-26; l'interdiction n'est toutefois formulée qu'en direction des linguistes). Il faut bien avouer cependant que, dans l'océan des opinions formulées sur le problème, elle est loin d'être la plus fréquente. J'en trouve fort peu de traces, par exemple, dans le livre de Hombert de 2005, et, si j'ai bien lu, aucune dans les travaux de Dessalles. La norme, à l'époque de Renan et de Saussure, puis au XXème siècle et, à ce qu'il me semble, encore plus au XXIème, c'est, au contraire de Saussure, de poser comme une nécessité absolue d'aborder le problème de l'origine du langage, c'est-à-dire celui du passage d'une éventuelle société humaine non pourvue du langage à une société qui s'en est pourvue.

Dans la masse innombrable des exemples possibles parmi les tenants de cette position, je n'en prendrai qu'un : celui de l'illustre monstre dicéphale qu'ont constitué, entre les deux guerres, Jacques Damourette et Edouard Pichon. Couple singulier : un vieil oncle presque infirme, passionné de langue française, un jeune neveu atteint très jeune d'une maladie inguérissable, médecin de son état, puis analyste et, aux approches de sa mort précoce, Président de la Société de Psychanalyse de Paris où il accueille, en 1938, un « des esprits les plus brillants de la jeune génération psychiatrique française » : Jacques Lacan, " encore tout florissant de bel âge ». Dès 1911, l'oncle et le neveu entreprennent la rédaction d'un vaste ouvrage qu'ils réussiront, en dépit de leurs maladies, à mener presque à son terme et à publier, encore partiellement. Il finira, après leur mort, par atteindre la masse de sept volumes in-quarto : Des mots à la pensée, Essai de grammaire de la langue française. 
Que diable vient faire le problème de l'origine du langage dans ce traité de grammaire française ? Les auteurs s'en expliquent avec la force et la lucidité qui leur sont habituelles :

« Il est impossible, même dans une grammaire spécifiquement française, de ne pas se poser le problème de la définition des traits constitutifs du langage en général, et par conséquent celui de l'origine dudit langage » (1927, tome I, § 46, p. 67).

Le par conséquent qui impose l'étude de l'origine comme implication de celle des traits constitutifs marque l'opposition à Saussure. Nos deux auteurs connaissent le $C L G$ depuis sa publication, en 1916, et ils partagent un grand nombre de ses vues théoriques. Mais pas celle qu'il professe sur l'origine du langage, non plus que celle - qui lui est liée - sur l'arbitraire du signe. Et ils s'engagent sans barguigner dans des spéculations diversement aventureuses, qui les amènent à une formulation dont ils se disent satisfaits :

« Nous pouvons dire, en une formule nette et satisfaisante, que le cri est devenu langage quand il a pris une valeur factive $»(1927$, tome $\mathrm{I}, \S 49$, p. 70$)$

La « valeur factive » ainsi conférée au « cri », et qui le fait passer au stade du langage, c'est celle qui permet au locuteur - je remarque au passage que Damourette et Pichon sont les inventeurs du mot et de la notion - de communiquer à l'allocutaire - autre innovation lexicale et conceptuelle des deux compères la représentation d'un fait.

Damourette et Pichon présentent leur théorie sur l'origine du langage de façon très, sans doute trop rapide : ce n'est tout de même pas l'essentiel de leur projet. Cependant leur théorie de la transformation du cri originel se retrouve dans de nombreux travaux. Roy Harris s'amuse beaucoup - enfin, je crois qu'il s'amuse - à énumérer, après Jespersen, les différents cris qui ont été utilisés pour caractériser les théories qui les ont cités. Il rappelle ainsi les théories bowwow, pooh-pooh, ding-dong, yo-he-ho, ta-ta, etc (1996, Introduction : vii). Je n'ai, je l'avoue, pas lu tous les textes mettant en œuvre ces belles théories. Je vois mal cependant comment elles échapperaient au double écueil constitué par les deux problèmes que nous avons aperçus plus haut à propos de Renan : celui du lien posé comme indissoluble entre langage et voix - le cri, c'est la voix à l'état brut - et celui de la relation entre les mots et les choses.

Le seul problème, rassurez-vous, qui nous reste à traiter, c'est celui de la façon dont Saussure navigue entre ces deux écueils.

1. Le problème de la voix. En ce point les choses sont simples. En 1894, dans l'un des textes les plus fascinants qu'il ait écrits - une ébauche non terminée d'un article d'hommage à la mémoire de Whitney, qui venait de mourir - Saussure tient les propos suivants : 
«Whitney dit que les hommes se sont servis de la voix comme ils se seraient servis du geste ou d'autre chose, et parce que cela leur a semblé plus commode de se servir de la voix. Nous estimons que ces deux lignes, qui ressemblent à un gros paradoxe, [ apportent ] la plus juste idée philosophique qui ait jamais été donnée du langage » (2002 : 215 ; Saussure se réfère à l'édition anglaise du livre de Whitney traduit par lui en français, 1877)

On voit de quelle façon radicale Saussure sur ce point s'oppose tant à Renan qu'à Damourette et Pichon : l'utilisation de la voix n'a rien de naturel ni de nécessaire. Mais qu'on ne s'y trompe surtout pas : Saussure n'est pas en train de prendre position en faveur de l'origine gestuelle (ou graphique, ou autre encore) du langage : on sait que ça peut se faire, que ça s'est fait, et que ça continue à se faire. On trouvera, sur le mode ludique, un autre exemple dans Arrivé 2007. Saussure traite le problème de façon exactement inversée : il renvoie dos à dos toutes les substances - vocale, gestuelle, graphique, etc - susceptibles d'être appelées à manifester les langues. Selon les Sources manuscrites du Cours II, il posait explicitement le problème de « l'indifférence du moyen de production » :

«Est-il si nécessaire que la langue se prononce par l'organe vocal ? Non : les mots peuvent être transposés dans l'écriture. L'instrument n'y fait rien. Ainsi la comparaison de la langue avec un autre système de signes nous permet d'aller jusque là, d'affirmer que là n'est pas l'essence de la langue » (Godel, 1957-1969 : 193-194 ; Engler, 1968-1989: 270).

J'insiste sur le dernier segment de ce passage : « là n'est pas l'essence de la langue ». L'adverbe là anaphorise le circonstanciel antécédent par l'organe vocal. Ce n'est pas l'utilisation de cet organe qui fait la spécificité de la langue.

Dans un autre passage, Saussure regrette le « mélange » qui est fait entre « qui est relatif à la voix et ce qui n'est relatif qu'à la traduction de la pensée par un signe qui peut être absolument quelconque et comporter un perfectionnement et une grammaire aussi bien selon des signes visuels ou tactiles que selon les signes non moins conventionnels qu'on choisira dans la voix » $(2002: 257)$.

S'ensuit, dans le Cours, la définition du signifiant comme « incorporel » et la belle démonstration de cette incorporalité qui est constituée par la mise en place de la notion de valeur : elle est exemplifiée, à la fois et sur le même plan, par l'exemple du phonème $r$ et de la lettre $t$. Les diverses réalisations accidentellement différentes qui peuvent être conférées à ces deux signifiants, l'un vocal, l'autre graphique, ne les empêchent en rien de conserver la même valeur. La relation entre les deux types de signifiants à 
l'égard de la langue qu'ils ont à manifester est exactement la même. On sait que la glossématique de Louis Hjelmslev et Hans Jørgen Uldall retiendra ce point de doctrine.

On vient d'apercevoir l'intérêt de cette analyse à l'égard de la mise en place de la notion fondamentale de valeur. On devine l'importance qu'elle va prendre à l'égard du problème de l'origine du langage : elle va permettre, très économiquement, d'évacuer toutes les spéculations incertaines sur les problèmes physiologiques posés par la production de la voix, par exemple les ténébreuses histoires de position haute ou basse du larynx ou de diamètre du canal hypoglosse.

2. Les mots et les choses. Faut-il le dire ? Saussure ne met évidemment pas en doute cette évidence : les mots permettent de désigner les choses du monde. Pas seulement les choses, précise-t-il : il s'élève contre la tradition qui consiste à n'examiner le « sens » des mots que par l'exemple des noms :

« C'est un accident quand le signe linguistique se trouve correspondre à un objet défini pour les sens, comme un cheval, le $\mathrm{feu}$, le soleil, plutôt qu'à une idée comme $\varepsilon \theta \eta \kappa \varepsilon$ " il posa ". Quel que soit l'importance de ce cas, il n'y aucune raison évidente, bien au contraire, de le prendre comme type du langage » $(2002: 230)$.

Ce qu'il met en cause de façon constante, c'est le régime adamique admis, à ce qu'il pense, par la quasi-totalité des "philosophes du langage »- de la relation entre les mots et les choses. C'est ce qu'il rejette sous le nom de nomenclature. Et pour rejeter cette conception du langage, il fait de nouveau intervenir, dans une note restée très lacunaire, le personnage d'Adam :

« Le problème du langage ne se pose à la plupart des esprits que sous la forme d'une nomenclature. Au chapitre IV de la Genèse, nous voyons Adam donner des noms [ ] et si [ ] on peut dire que [ ]» $(2002: 327$; Saussure se réfère de façon inexacte au chapitre IV de la Genèse ; c'est au chapitre II qu'intervient la nomination des êtres vivants).

Je retiens particulièrement ce passage, dénommé, sans excès d'indulgence, Catalogue des erreurs fondamentales :

«Méritent le nom d'erreurs fondamentales à cause de leurs conséquences :

L'erreur des signes individuels pris chacun pour soi :

- ou erreur de croire qu'une langue composée de 500 mots représente 500 signes +500 significations,

- ou erreur de croire qu'on représente en rien le phénomène de la langue quand on se croit autorisé à dire " le mot et sa signification ", oubliant que le mot est entouré de [ ] $\quad$ (2002:106). 
Saussure, en ce point, garde une fois de plus le silence. C'est ce qu'il fait souvent quand il atteint les aspects les plus importants de son appareil théorique, ici la définition du mot comme terme d'un système. Mais il lui arrive parfois d'expliciter sa pensée jusqu'à lui donner une portée très générale :

« Si un objet pouvait, où que ce soit, être le terme sur lequel est fixé le signe, la linguistique cesserait instantanément d'être ce qu'elle est, depuis le sommet jusqu'à la base ; du reste l'esprit humain du même coup » $(2002: 230)$

On ne saurait mettre en cause de façon plus déterminée toute conception « nomenclaturiste » du langage. Et on ne s'étonne pas de constater que le projet d'ouvrage « De l'essence double du langage » se termine par une spéculation très provocatrice sur une langue qui ne comporterait que deux signes :

« Ainsi, dans une langue composée au total de deux signes, $b a$ et la, la totalité des perceptions confuses de l'esprit viendra NECESSAIREMENT se ranger ou sous $b a$ ou sous la. L'esprit trouvera, du simple fait qu'il existe une différence ba/la et qu'il n'en existe pas d'autre, un caractère distinctif lui permettant régulièrement de tout classer sous le premier ou sous un des deux chapitres (par exemple la distinction de solide et de non solide) $»(2002: 88)$

Saussure, on l'a vu, a un vif penchant pour le silence. Au point qu'on peut se demander si le silence n'est pas un élément central de son appareil théorique. Je vais bientôt suivre son exemple, vous laissant le soin de réfléchir dans le même silence que lui et que moi sur ce que sa théorie, incontestablement très paradoxale, voire provocatrice, de l'origine du langage apporte à la conception du langage. Avant de me taire, j'attirerai toutefois votre attention sur ce qui me paraît être deux évidences :

1. La conception non-référentielle et systémique du langage qui est ainsi continûment mise en place a pour conséquence immédiate d'éliminer jusqu'à la possibilité de ressemblance entre les mots et les choses. C'est ainsi que s'institue et se justifie le principe fondamental de l'arbitraire du signe, et, implication à vrai dire annexe, mais importante dans l'histoire des idées sur le langage, l'élimination absolue de la possibilité même de l'onomatopée et, d'une façon générale, de l'analogie, quand on la prend dans le sens de relation analogique entre référent et/ou signifié et signifiant.

2. La position spécifique de Saussure sur l'origine du langage et des langues est totalement inséparable de l'entier de sa conception du langage. Tient- 
on à mettre en cause cette position ? C'est un geste parfaitement légitime. Mais il faut en tirer la conséquence immédiate : renoncer entièrement à l'appareil notionnel saussurien.

\section{BIBLIOGRAPHIE}

Abel, Carl, 1885, « Über den Ursprung der Sprache », in Sprachwissenschaftliche Abhandlungen, Leipzig : 285-309.

Arrivé, Michel, 2007, Á la recherche de Ferdinand de Saussure, Paris, PUF. Arrivé, M., 2008, Le linguiste et l'inconscient, Paris, PUF.

Bergounioux, Gabriel, 2005, "L'origine du langage : mythes et théories », in Hombert (éd.) 2005 : 14-39.

Damourette, Jacques et Pichon Edouard, sd [1927], Des mots à la pensée. Essai de grammaire de la langue française, tome I, Paris, d'Artrey.

Dessalles, Jean-Louis, 2000, Aux origines du langage : une histoire naturelle de la parole, Paris, HERMES Science.

Dessalles, J.-L., 2006, « Du protolangage au langage : modèle d'une transition ", Marges lingustiques, 11, mai : 142-152.

Engler, Rudolf, 1968-1989, Ferdinand de Saussure, Cours de linguistique générale, Edition critique, Wiesbaden, Otto Harrassowitz.

Godel, Robert, 1957-1969, Les sources manuscrites du Cours de linguistique générale, Genève, Droz.

Harris, Roy, (éd.), 1996, The origin of language, Bristol, Thoemmes Press.

Henry, Victor, 1896-sd, Antinomies linguistiques, Félix Alcan, puis Didier Eruditions.

Hombert, Jean-Marie (éd.), 2005, Aux origines des langues et du langage, Paris, Fayard.

Renan, Ernest, 1848-1958-sd, De l'origine du langage, Paris, Calmann-Lévy, puis Didier Érudition.

Robert, Thomas, 2010, « Saussure et l'origine du langage. Un interdit à dépasser par la philosophie linguistique », Rivista italiana di filosofia del linguaggio, $3: 147-156$.

Saussure, Ferdinand de, 1916-1922-1986, Cours de linguistique générale, Paris, Payot.

Saussure, F. de, 2002, Écrits de linguistique générale, Paris, Gallimard.

Saussure, F. de, voir aussi Engler et Godel.

Sperber, Hans, 1912, « Über den Einfluss sexueller Momente auf Enstehung und Entwicklung der Sprache », Imago, 1, $5:$ 405-453.

Whitney, William Dwight, 1877-1977, La vie du langage, Paris, GermerBaillière et Cie, puis Didier érudition. 
MICHEL ARRIVE,

PARIS OUEST NANTERRE

UMR MODYCO. 